\title{
The effect of anisotropic flux pinning microstructure on the sample length dependence of the magnetization critical current density in niobium-titanium superconductors
}

\author{
C. Bormio Nunes, ${ }^{a)}$ R. W. Heussner, ${ }^{\text {b) }}$ and D. C. Larbalestier ${ }^{\text {b) }}$ \\ Applied Superconductivity Center, University of Wisconsin-Madison, 1500 Engineering Drive, Madison, \\ Wisconsin 53706
}

(Received 5 March 1996; accepted for publication 3 May 1996)

\begin{abstract}
Magnetization measurements of the critical current density $J_{c}$ in $\mathrm{Nb} 47 \mathrm{wt} \% \mathrm{Ti}$ with $\mathrm{Nb}$ artificial pinning centers revealed that the shape and magnitude of the field dependent magnetization hysteresis $\Delta M(H)$ was a strong function of the sample length and that $\Delta M(H)$ for short wire samples was up to six times smaller than for long wires. This is caused by the strong anisotropy of the critical current density $J_{c}$. The magnitude of $J_{c}$ flowing perpendicular to the wire axis $J_{\perp}$ was deduced to be 50-175 times smaller than the longitudinal current density $J_{\|}$. The source of the anisotropy lies in the anisotropic flux pinning microstructure of the wires. When the magnetization current crosses perpendicular to the filament axis at each end of the wire, the Lorentz force is parallel to the pinning center axis. The pinning force is weak in this direction and $J_{\perp}$ is correspondingly small. The technologically important critical current density is the longitudinal current density $J_{\|}$. It can be extracted from magnetization measurements only in the case of large length to diameter filaments, as is quantitatively analyzed here. (C) 1996 American Institute of Physics. [S0021-8979(96)09715-0]
\end{abstract}

\section{INTRODUCTION}

Artificial pinning center (APC) $\mathrm{Nb}-\mathrm{Ti}$ composite designs have produced significant improvements in the critical current density $J_{c}$ in magnetic fields of up to $5 \mathrm{~T}$, as compared to conventionally processed composites. ${ }^{1-3}$ However, improvements at higher fields have been impeded by the fact that the upper critical magnetic field $H_{c 2}$ is significantly depressed in virtually all existing APC composites ${ }^{1-3}$ and because the bulk pinning force $F_{p}$ curves tend to peak at lower values of the reduced field $\left(h=H / H_{c 2}\right), h \approx 0.3$ vs $h \approx 0.5$, than is the case for conventional composites.

In order to better understand the attainable $J_{c}$ values of APC wires, we decided to study the magnetic field and temperature dependent bulk pinning force density curves $F_{p}(H, T)$ over the whole field range up to $H_{c 2}$. We used a vibrating sample magnetometer (VSM) which permits continuous magnetization hysteresis $\Delta M(H, T)$ measurements from 0 to $14 \mathrm{~T}$ and from 2 to $10 \mathrm{~K}$. We initially assumed that we could extract $J_{c}$ and $F_{p}$ from $\Delta M(H, T)$ measurements using the conventional Bean model analysis. ${ }^{4}$ However, we found that the magnetization $J_{c}$ derived in this way had a very different field dependence than the measured transport $J_{c}$ in several cases. Being concerned about the effect that the anisotropy of the flux pinning microstructure in APC wires might play in determining the magnetization, we made systematic measurements of the hysteretic magnetization $\Delta M(H)$ as a function of sample length.

The issue of the sample-length dependence of the magnetization in various types of superconducting composites and the $J_{c}$ derived from such measurements has been addressed in several recent studies. A length dependence of

\footnotetext{
a) On leave from FAENQUIL/DEMAR, 12600-000 Lorena-SP, Brazil.

${ }^{b)}$ Materials Science Program.
}

$\Delta M(H)$ for multifilamentary $\mathrm{Nb}-\mathrm{Ti}$ wires containing very fine filaments was observed for conditions under which there was a proximity-effect coupling across the interfilament copper. ${ }^{5}$ The effect was also noted in a study of $\mathrm{Nb}_{3} \mathrm{Sn}$ wires in which there was significant filament-to-filament touching, as frequently occurs in wires made by the internal-tindiffusion process. ${ }^{6}$ It is also well-attested that the orientation of the pinning centers and the magnetic field axis plays a large role in conventionally processed, two-phase $\mathrm{Nb}-\mathrm{Ti}$ wires which are flattened from round to rectangular. ${ }^{7-9} \mathrm{Mag}-$ netization measurements of the longitudinal and circumferential critical current densities of round wires also showed evidence for a $J_{c}$ anisotropy. ${ }^{10}$ These studies on conventional $\mathrm{Nb}-\mathrm{Ti}$ reported anisotropy ratios of up to 8-12.

The present work establishes the experimental conditions under which the hysteretic magnetization of superconductors with anisotropic pinning center arrays can be correctly measured and the technologically important longitudinal critical current density $J_{\|}$extracted. The APC samples and the experimental details are described in Sec. II, and the magnetization measurements in Sec. III. In Sec. IV, the critical current density anisotropy $J_{\|} / J_{\perp}$ vs $H$ and the bulk pinning force $F_{p}\left(J_{\|}\right)$vs $H$ are calculated from the length dependent magnetization data, using an anisotropic Bean model for cylindrical shaped samples. ${ }^{11}$ Section V summarizes the work.

\section{EXPERIMENTAL DETAILS}

\section{A. APC wire design}

The APC composite was fabricated using the rod-based (RBAPC) process discussed previously. ${ }^{3}$ Thirty-one rods of $\mathrm{Nb}$ were arranged in a hexagonal array within a bundle of 96 rods of $\mathrm{Nb} 47 \mathrm{wt} \% \mathrm{Ti}$, resulting in a $24.4 \%$ pin volume fraction. The hexagonal stack was canned in copper, ex- 
TABLE I. The APC sample characteristics. $d_{p}$ is the nominal pinning center diameter, $d_{f}$ is the filament diameter, $L$ and $A_{f}$ are the filament length and aspect ratio. Three measurement techniques used: transport $J_{c t}$ and magnetization by VSM and SQUID magnetometers.

\begin{tabular}{|c|c|c|c|c|c|c|}
\hline & \multirow{2}{*}{$\begin{array}{c}d_{p} \\
(\mathrm{~nm})\end{array}$} & \multirow{2}{*}{$\begin{array}{c}d_{f} \\
(\mu \mathrm{m})\end{array}$} & \multicolumn{3}{|c|}{$L(\mathrm{~mm})$} & \multirow[b]{2}{*}{$A_{f}$} \\
\hline & & & $J_{c t}(4.2 \mathrm{~K})$ & VSM (4.2 K) & SQUID $(6 \mathrm{~K})$ & \\
\hline & & & 330 & & & 1400 \\
\hline & & & & 56 & & 240 \\
\hline \multirow[t]{6}{*}{ A } & 165 & 236 & & & 97 & 410 \\
\hline & & & & & 25 & 110 \\
\hline & & & & & 12 & 51 \\
\hline & & & & & 2.3 & 10 \\
\hline & & & 330 & & & 5000 \\
\hline & & & & 230 & & 3500 \\
\hline \multirow[t]{6}{*}{ B } & 46 & 66 & & & 157 & 2400 \\
\hline & & & & & 40 & 610 \\
\hline & & & & & 10 & 150 \\
\hline & & & & & 2.0 & 30 \\
\hline & & & 330 & & & 1850 \\
\hline & & & & 3 & & 17 \\
\hline \multirow[t]{4}{*}{$\mathrm{C}$} & 47 & 178 & & & 85 & 480 \\
\hline & & & & & 30 & 170 \\
\hline & & & & & 7.5 & 42 \\
\hline & & & & & 3.1 & 17 \\
\hline
\end{tabular}

truded to $4 \mathrm{~mm}$ diameter and cold drawn to $1.5 \mathrm{~mm}$ diameter. The wire was then cut into 127 pieces and the outer copper was etched away. The bare $\mathrm{Nb}-\mathrm{Ti} / \mathrm{Nb}$ filaments were restacked, clad in $\mathrm{Cu}$, and extruded for a second time. The restacking and extrusion process was repeated a third time, producing a monofilament containing $5 \times 10^{5}$ pinning centers. Some of the third extrusion wire was drawn to $0.08 \mathrm{~mm}$ diameter (nominal pin diameter $40 \mathrm{~nm}$ ), while the rest of the wire was incorporated into a fourth extrusion composite which produced nominal pin diameters down to $15 \mathrm{~nm}$.

Table I presents the characteristics of the three different APC wires used for this study including the nominal pin diameter $d_{p}$, the filament diameter $d_{f}$, the length $L$, and the filament aspect ratio $A_{f}$. The nominal pin diameter is defined as

$$
d_{p}=\frac{d_{w}}{\sqrt{N(1+R)}},
$$

where $d_{w}$ is the wire diameter, $N$ is the number of stacked rods (i.e., $127^{3}$ for the third extrusion wires $A$ and $B$ and $127^{3} \times 7$ for the fourth extrusion wire C) and $R$ is the copperto-superconductor volume ratio. It should be noted, as discussed in detail in Ref. 3, that the actual pin thickness at the wire sizes discussed in this paper was several times smaller than $d_{p}$ because the round pins flatten into ribbons whose aspect ratio increases with decreasing pin size. However, the pins appear to be continuous over macroscopic lengths, probably the whole length of each wire. Thus, the flux pinning microstructure produced by the fabrication process is extremely anisotropic.

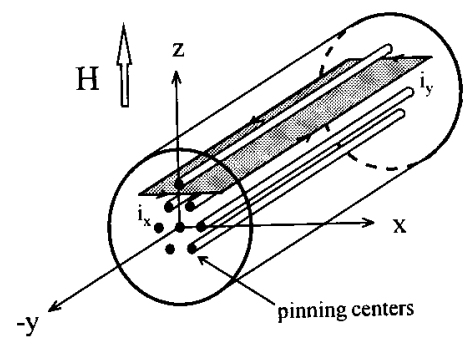

FIG. 1. Schematic drawing of an APC filament showing the positions of the pins and the magnetization induced currents in a magnetic field applied normal to the wire axis.

\section{B. Transport $\boldsymbol{J}_{c}$ measurements}

The transport critical current, $I_{c}$, was measured with voltage taps spaced 22 or $33 \mathrm{~cm}$ apart, using a resistivity criterion of $10^{-14} \Omega \mathrm{m}$. Samples were tested in liquid helium at $4.2 \mathrm{~K}$ with the magnetic field applied normal to the wire axis. The transport critical current density $J_{c t}$ was determined by dividing the $I_{c}$ by the whole $\mathrm{Nb}-\mathrm{Ti}$ and Nb crosssectional area. This measurement yields the longitudinal critical current density $J_{\|}$.

\section{Magnetization measurements}

Magnetic moment measurements were made using vibrating sample (VSM) and SQUID magnetometers, calibrated with transverse wire samples (aspect ratio $\approx 10: 1$ ) of $\mathrm{Ni}$ and/or Pd. The VSM and SQUID instruments have usable sample space diameters $d_{b}$ normal to the applied field of 3 and $5 \mathrm{~mm}$, respectively. All measurements were made with the applied magnetic field perpendicular to the wire axis so that current was induced to flow along the length of the filament and to return by crossing the filament at each end of the wire. Since samples A, B and C were monofilament samples, there is no possibility of current crossing the copper sheath. In order to test the effect of sample length on $\Delta M$, two types of sample configuration were used. For the case of $L<d_{b}$, many straight wire pieces were used in order to obtain a large signal. For $L>d_{b}$, the wire was wound into an open coil with an outer diameter of just less than $d_{b}$. VSM magnetization data were taken continuously in the field range from 0 to $12 \mathrm{~T}$ using a ramp rate of $0.009-0.03 \mathrm{~T} / \mathrm{s}$, the slower rates being used near $H_{c 2}$. The temperature was fixed at $4.2 \mathrm{~K}$ with an allowed variation of $50 \mathrm{mK}$. The magnetization critical current density $J_{\mathrm{cm}}$ was determined from the VSM magnetization measurements using the Bean model for a cylinder in perpendicular field ${ }^{4}$

$$
J_{\mathrm{cm}}(H, T)=\frac{3 \pi \Delta M(H, T)}{4 d_{f}},
$$

where $\Delta M$ is the width of the magnetization hysteresis and $d_{f}$ is the filament diameter. SQUID magnetization data were taken from 0 to $5 \mathrm{~T}$ in $0.2 \mathrm{~T}$ increments. The samples were measured at $6 \mathrm{~K}$, a temperature for which the $H_{c 2}$ values of the wires were slightly higher than $5 \mathrm{~T}$.

Figure 1 shows a sketch of the APC filament. The field was applied in the $z$ direction, normal to the wire axis, inducing magnetization currents to flow in the $x y$ plane. The 
directions of the magnetization induced currents with respect to the pinning centers are shown. The orthogonal portions of the complete current loop $I^{x}$ and $I^{y}$ lie along the $x$ and $y$ directions and are normal and parallel, respectively, to the longitudinal axes of the pinning centers. Two orthogonal Lorentz forces $F_{L}^{x}$ and $F_{L}^{y}$ originate from the interaction between the magnetic flux vortices and the magnetizationinduced currents $I^{y}$ and $I^{x}$, respectively. For the case where the current flows parallel to the filament, the vortices are forced $\left(F_{L}^{x}\right)$, normal to the long axis of the pinning centers such that the pins act as effective obstacles to vortex motion. On the other hand vortices are forced to move parallel to the pins $\left(F_{L}^{y}\right)$ for the transverse components of the current and the pins do not then act as significant obstacles.

The dominant flux pinning interaction mechanism between vortices and pinning centers in $\mathrm{Nb}-\mathrm{Ti}$ superconductors is still debated. ${ }^{12-15}$ Traditionally a core pinning interaction, has been used to explain flux pinning in conventional ${ }^{16,17}$ and $\mathrm{APC}^{12} \mathrm{Nb}-\mathrm{Ti}$. However, magnetic interactions ${ }^{18-20}$ have been more recently invoked to describe the elementary pinning interaction in both APC and conventional $\mathrm{Nb}-\mathrm{Ti}^{21}{ }^{21}$ Regardless of the type of interaction between vortices and pinning centers, the fact that the flux pinning force is larger in the $x$ direction than in the $y$ direction gives rise to the critical current density anisotropy, $J_{c}^{x}<J_{c}^{y}$. Throughout the rest of the paper, $J_{c}^{x}$ and $J_{c}^{y}$ will be designated as $J_{\perp}$ and $J_{\|}$, respectively.

\section{RESULTS}

Figures 2(a)-2(c) compare the $F_{p}(H)$ curves derived from the transport $J_{c}$ measurements to those derived from VSM magnetization for wires $\mathrm{A}, \mathrm{B}$, and $\mathrm{C}$ at $4.2 \mathrm{~K}$. The shapes of curves A and B [Figs. 2(a) and 2(b)] are similar although the magnitude of the transport $F_{p}$ is slightly lower. However, sample $\mathrm{C}$ exhibits transport and magnetization $F_{p}(H)$ curves which have remarkably different shapes and magnitudes. The peak transport $F_{p}$ of $20 \mathrm{GN} / \mathrm{m}^{3}$ occurs at 3 $\mathrm{T}$ while the peak magnetization $F_{p}$ occurs at $5 \mathrm{~T}$ and is only $7 \mathrm{GN} / \mathrm{m}^{3}$. The very large difference for sample $\mathrm{C}$ was our first indication that $\Delta M$ is a strong function of sample length or, more precisely, to the filament length-to-diameter aspect ratio, $A_{f}$. The filament aspect ratios of the samples $\mathrm{A}, \mathrm{B}$, and C reported in Fig. 2 are 240, 3500, and 17, respectively. As can be observed from Fig. 2, the agreement between the transport and magnetization $F_{p}(H)$ curves increases with increasing $A_{f}$. Figure 2 clearly shows that the $J_{c}$ extracted from $\Delta M(H)$ measurements made on wires in a perpendicular field approximates the transport $J_{c}$ only for sufficiently large $A_{f}$. These initial results motivated us to make additional magnetization measurements designed to study the $\Delta M(H)$ vs $A_{f}$ relationship explicitly.

To clarify the $\Delta M(H)$ versus length relationship, more systematic experiments were made using a SQUID magnetometer. Four different lengths of each wire were measured, such that the longest wire in each set was 30-80 times longer than the shortest wire. The sample details are given in Table I. Figures 3(a)-3(c) show the measured $\Delta M(H)$ as a function of the applied field for A, B, and C. All three samples show a strong $\Delta M(H)$ dependence on the sample length,
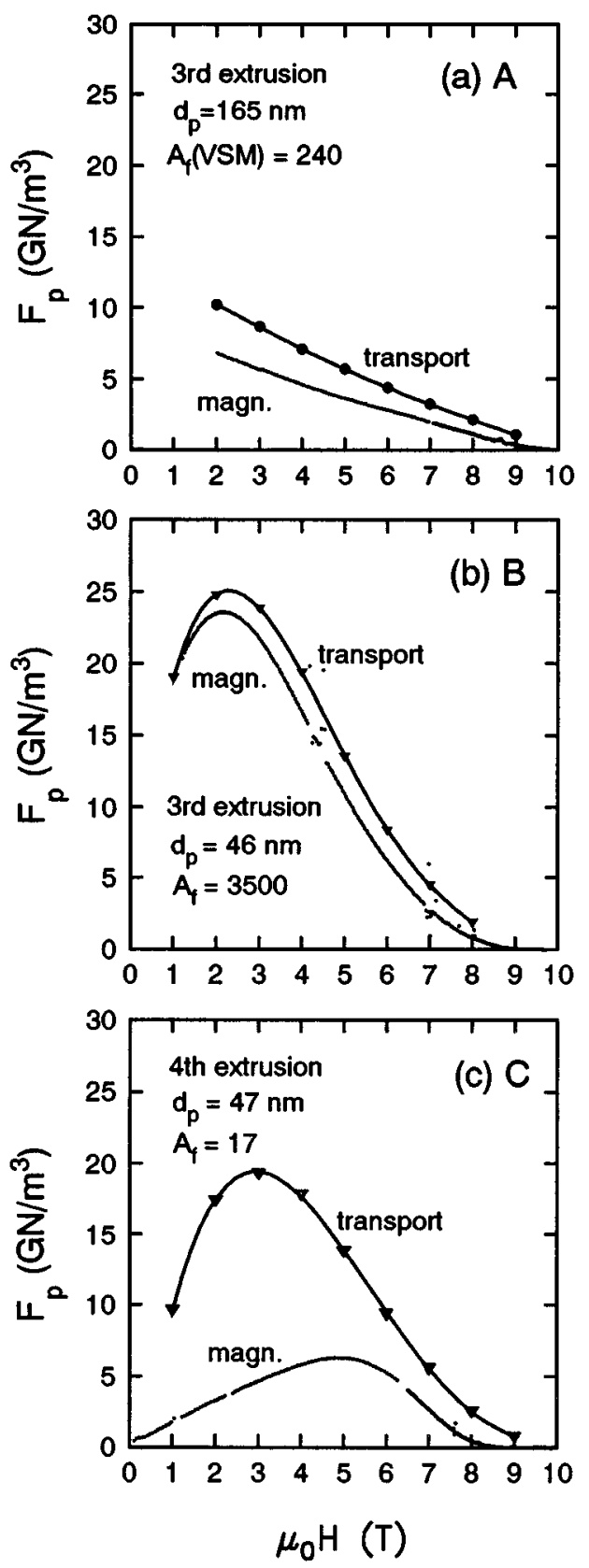

FIG. 2. $F_{p}$ vs $\mu_{0} H$ curves comparing magnetization and transport data taken at $4.2 \mathrm{~K}$ for samples (a) A, (b) B, and (c) C. The transport samples were 60 $\mathrm{cm}$ long. The magnetization samples had wire aspect ratios $\left(A_{f}=1 / d_{f}\right)$ of 240, 3500, and 17 for samples A, B, and C, respectively.

suggestive of the effect of critical current density anisotropy. Section IV describes an anisotropic critical current model that was used to extract $J_{\|}$and $J_{\perp}$ values of the APC wires from the length-dependent magnetization measurements.

\section{ANISOTROPIC CRITICAL CURRENT DENSITY BEAN MODEL}

Gyorgy et al., ${ }^{22}$ and Sauerzopf et al.,${ }^{23}$ calculated the dependence of the magnetization on the anisotropic current critical densities $J_{c}^{(a)}$ (current flowing parallel to side $a$ ) and $J_{c}^{(c)}$ (parallel to side $c$ ) of thin parallelpiped-shaped samples of dimensions $a$ and $c$ placed in an applied field normal to 

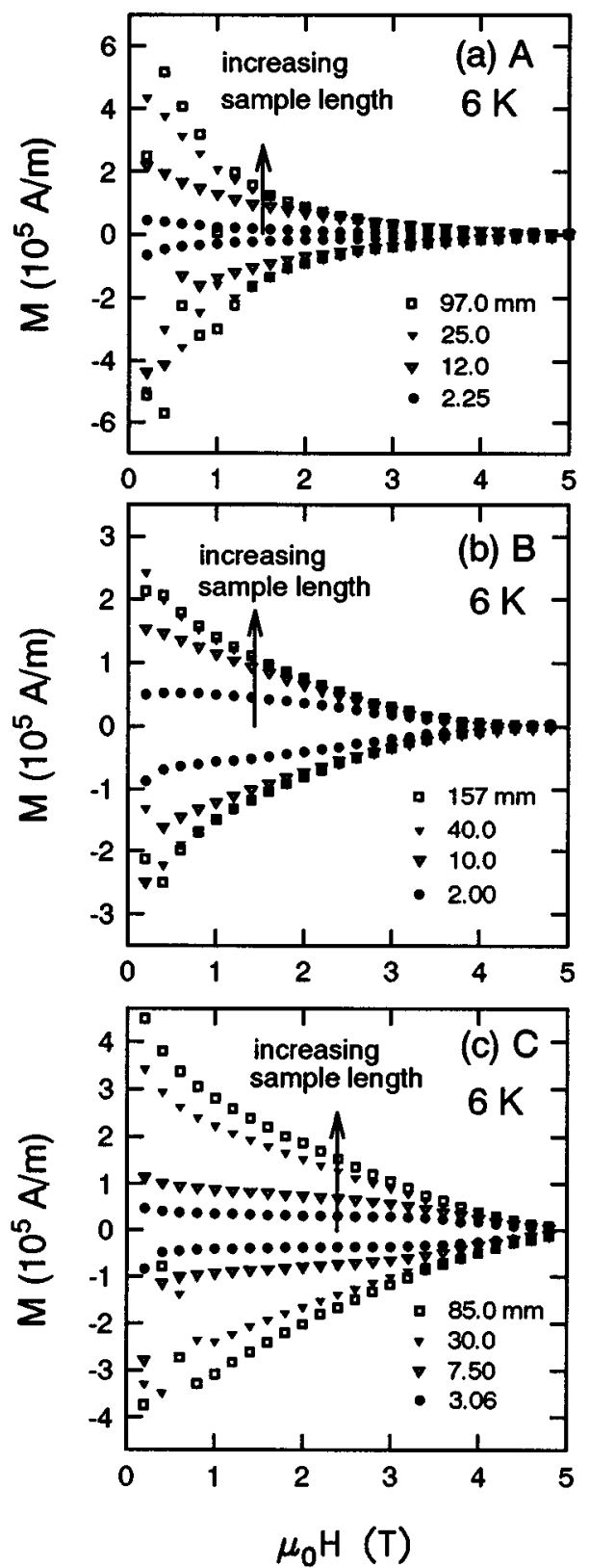

FIG. 3. $M$ vs $\mu_{0} H$ at $6 \mathrm{~K}$ for samples (a) A, (b) B, and (c) C as a function of sample length.

the $a c$ plane. More recently, Sumption, ${ }^{11}$ derived an equivalent model for cylindrical-shaped samples in a field applied normal to the cylinder axis. If $J_{\|}$and $J_{\perp}$ are the critical current densities in the direction parallel and perpendicular to the cylinder axis, respectively, and $L$ and $R$ are the cylinder length and radius, then $\Delta M$ is given by

$$
\Delta M(H)=\frac{8 J_{\|} R}{3 \pi}\left(1-\frac{3 \pi R}{16 L} \frac{J_{\|}}{J_{\perp}}\right)
$$

when

$$
\begin{aligned}
& \frac{J_{\perp}}{J_{\|}}>\frac{2 R}{L}, \\
& \Delta M(H)=\frac{J_{\perp} L}{2}\left(1-\frac{2 L}{3 \pi R} \frac{J_{\perp}}{J_{\|}}\right)
\end{aligned}
$$

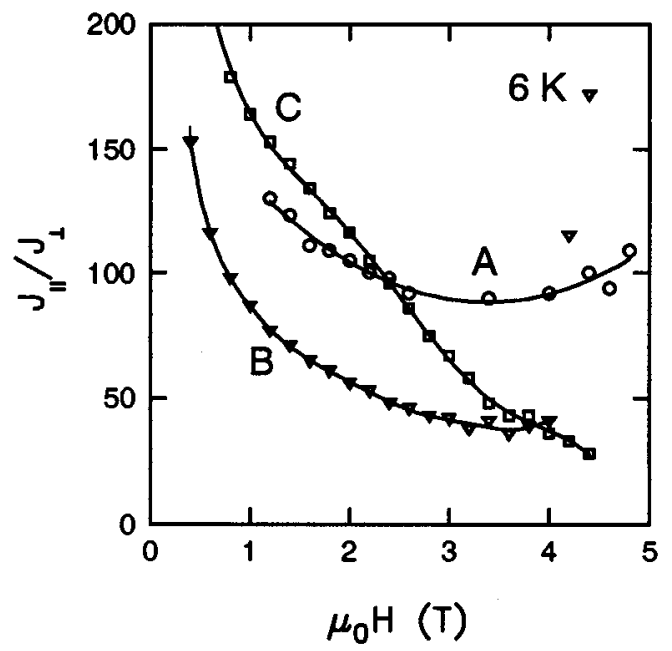

FIG. 4. $J_{\|} / J_{\perp}$ vs $\mu_{0} H$ for samples A, B, and C as calculated from the length dependent $\Delta M$ measurements using an extended Bean model for the anisotropic critical current density (see Ref. 11).

when

$$
\frac{J_{\perp}}{J_{\|}}<\frac{2 R}{L} .
$$

Equations (3) and (4) are in MKS units and $\Delta M(H)$ is the width of the magnetization hysteresis.

$J_{\|}$and $J_{\perp}$ were determined for each wire at each applied field by fitting Eqs. (3) and (4) to the four $\Delta M(L)$ data points for each field. The $J_{c}$ anisotropy curves of Fig. 4 were constructed from these calculated $J_{\|}$and $J_{\perp}$ values. Figure 4 shows that the anisotropy ratio $J_{\|} / J_{\perp}$ vs $H$ varies from $\sim 40$ to 175 . The anisotropy ratio appears to decrease with decreasing pin size, as is made clear by comparing wires A and $\mathrm{B}$, which are both third extrusion wires, differing only in their value of $d_{p}, 165$, and $46 \mathrm{~nm}$, respectively. It appears that the field dependence of the anisotropy increases as pin size is decreased, consistent with a recent magnetic pinning model. $^{21}$

The anisotropy ratio for $\mathrm{C}$ also decreases, but in a nearly monotonic way, as the applied field increased to $H_{c 2}$. Sample $\mathrm{C}$ comes from a fourth extrusion composite and, in principle, should have the same properties as sample B because it has the same nominal pin diameter of $46 \mathrm{~nm}$ and because TEM analysis of both composite nanostructures showed that the pin thickness and distribution are similar. ${ }^{24}$ However, earlier transport $J_{c}$ measurements ${ }^{3}$ have shown that samples B and $\mathrm{C}$ did not exhibit the same $J_{c}(H)$ behavior. As suggested there, the difference is probably due to a change in the chemical homogeneity of the pins due to additional interdiffusion introduced during the fourth warm extrusion step.

Figure 5 shows the bulk pinning force $F_{p}\left(J_{\|}\right)$vs $H$ curves derived from the longitudinal component of the data in Fig. 3 using the anisotropic Bean model. The $F_{p}\left(J_{\|}\right)$vs $H$ curve shapes are now indeed very similar to the transport $F_{p}(H)$ curves of Fig. 2. However, an exact comparison of either the shape or the magnitude of $F_{p}$ cannot be made, since the data were taken at different temperatures and there is not good temperature scaling in this proximity-effect- 


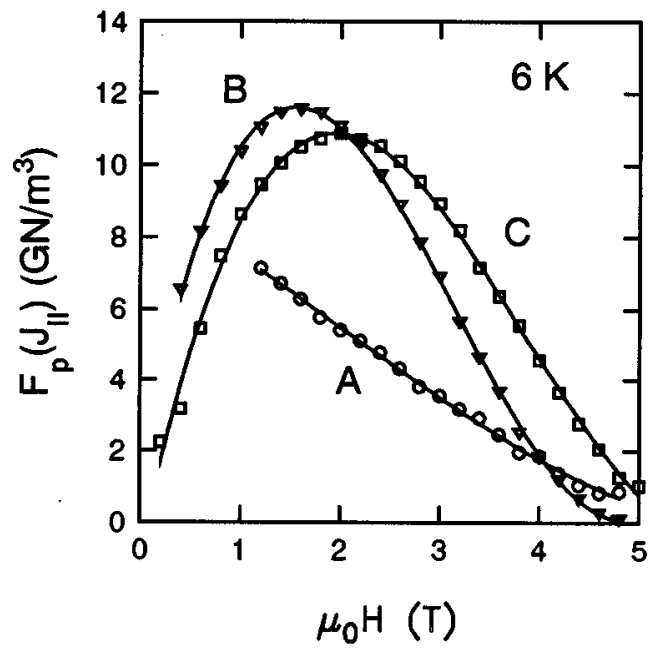

FIG. 5. $F_{p}\left(J_{\|}\right)$vs $\mu_{0} H$ samples $\mathrm{A}, \mathrm{B}$, and $\mathrm{C}$ as derived from the length dependent $\Delta M$ measurements using an extended Bean model with anisotropic critical current density (see Ref. 11).

coupled system. ${ }^{17,21}$ The general agreement between the shapes of the two curve sets is certainly consistent with predictions of the anisotropic Bean model. It is also possible to extract the perpendicular critical current density, $J_{\perp}$, which crosses the pinning centers. This information may help understand the proximity-effect coupling which occurs between the $\mathrm{Nb}-\mathrm{Ti}$ matrix and artificial pinning centers, ${ }^{21}$ as we will explore elsewhere.

\section{SUMMARY}

The sample-length dependence of the hysteretic magnetization in APC and other wires having a strongly anisotropic pinning microstructure appears to be a new result, for it is neither related to proximity-effect coupling between the superconductor and the normal metal matrix as is the case for $\mathrm{Nb}-\mathrm{Ti}$ multifilamentary strand with small interfilament spacing nor to interfilament contact, as is the case for some $\mathrm{Nb}_{3} \mathrm{Sn}$ strands because, in the present case, large $(>60 \mu \mathrm{m}$ diam) monofilament wires were measured. Therefore, we conclude that the observed results are determined solely by the anisotropy of the flux pinning microstructure. The results show that the technologically important value of the critical current density $J_{\|}$can be calculated from magnetization measurements using a conventional Bean model analysis only when the filament aspect ratio $A_{f}$ is large enough so that $\Delta M(L)$ saturates. The characteristic value of $A_{f}$ for which $\Delta M$ saturates depends on the characteristics of the flux pinning anisotropy. However, the anisotropic Bean model of Sumption appears to permit an explicit separation of the longitudinal and transverse critical current densities. The calculated $J_{\|} / J_{\perp}$ values varied from 40 to 175 for the APC wires studied here. These anisotropy values are large when compared to the values of 8-12 reported for conventionally processed $\mathrm{Nb}-\mathrm{Ti}$ wires.

\section{ACKNOWLEDGMENTS}

We would like to thank João Vargas and Alex Gurevich for useful discussions and Alex Squitieri for his technical assistance. Cristina B. Nunes was supported by a fellowship from CNPq-Brasília/Brazil. This work was supported by the Department of Energy-Division of High Energy Physics.

${ }^{1}$ K. Matsumoto, H. Takewasi, Y. Tanaka, O. Miura, K. Yamafuji, K. Funaki, M. Iwakuna, and T. Matsushita, Appl. Phys. Lett. 64, 115 (1994).

${ }^{2}$ L. R. Motowidlo, B. A. Zeitlin, M. S. Walker, and P. Haldar, Appl. Phys. Lett. 61, 991 (1992).

${ }^{3}$ R. W. Heussner, P. D. Jablonski, P. J. Lee, and D. C. Larbalestier, IEEE Trans. Appl. Supercond. 5, 1705 (1995).

${ }^{4}$ C. P. Bean, Rev. Mod. Phys. 36, 31 (1964).

${ }^{5}$ M. D. Sumption and E. W. Collings, Cryogenics 34, 491 (1994).

${ }^{6}$ R. B. Goldfarb and K. Itoh, J. Appl. Phys. 75, 2115 (1994).

${ }^{7}$ K. J. Best, D. Genevey, H. Hillmann, L. Krempasky, M. Polak, and B. Turck, IEEE Trans. Magn. 15, 395 (1979).

${ }^{8}$ K. J. Best, D. Genevey, H. Hillmann, L. Krempasky, M. Polak, and B. Turck, IEEE Trans. Magn. 15, 765 (1979).

${ }^{9}$ L. D. Cooley, P. Jablonski, P. J. Lee, and D. C. Larbalestier, Appl. Phys. Lett. 58, 2984 (1991).

${ }^{10}$ H. Küpfer and T. Matsushita, J. Appl. Phys. 63, 5060 (1988).

${ }^{11}$ M. Sumption, Appl. Supercond. 2, 41 (1994).

${ }^{12}$ L. D. Cooley, P. J. Lee, and D. C. Larbalestier, IEEE Trans. Magn. 27, 1096 (1991).

${ }^{13}$ C. Bormio Nunes and D. G. Pinatti, Physica B 194-196, 1817 (1994).

${ }^{14}$ A. I. Larkin and Yu. N. Ovchinnikov, J. Low Temp. Phys. 34, 409 (1979).

${ }^{15}$ R. G. Hampshire and M. T. Taylor, J. Phys. F 2, 89 (1972).

${ }^{16}$ T. Matsushita and H. Küpfer, J. Appl. Phys. 63, 5048 (1988).

${ }^{17}$ C. Meingast and D. C. Larbalestier, J. Appl. Phys. 66, 5971 (1989).

${ }^{18}$ C. P. Bean and J. D. Jones, Phys. Rev. Lett. 12, 14 (1964).

${ }^{19}$ X. G. Qui, S. Takahashi, and M. Tachiki, Physica C 216, 49 (1993).

${ }^{20}$ A. Gurevich and L. D. Cooley, Phys. Rev. B 13563 (1994).

${ }^{21}$ L. D. Cooley, P. J. Lee, and D. C. Larbalestier, Phys. Rev. B 53, 6638 (1996).

${ }^{22}$ E. M. Gyorgy, R. B. von Dover, K. A. Jackson, L. F. Schneemeyer, and J. V. Waszczak, Appl. Phys. Lett. 55, 283 (1989).

${ }^{23}$ F. M. Sauerzopf, H. P. Wiesinger, and H. W. Weber, Cryogenics 30, 650 (1990).

${ }^{24}$ R. W. Heussner, C. Bormio Nunes, P. J. Lee, P. D. Jablonski, and D. C. Larbalestier, J. Appl. Phys. 80, 1640 (1996). 